SCIENTIFIC LETTER

\title{
Periodic breathing as a feature of right heart failure in congenital heart disease
}

\author{
P Georgiadou, S V Babu-Narayan, D P Francis, D T Kremastinos, M A Gatzoulis
}

Heart 2004;90:1075-1076. doi: 10.1136/hrt.2003.025221

A lthough periodic breathing (PB) has been previously reported as a feature of right heart failure, it has never been observed in any manifestation of adult congenital heart disease. We report two cases of patients with tetralogy of Fallot (ToF) demonstrating this phenomenon late after repair. Both patients had right heart failure because of pulmonary regurgitation (PR) assessed by cardiovascular magnetic resonance imaging and exhibited $\mathrm{PB}$ on a background of a depressed ventilatory drive, long circulatory time, and elevated sympathetic activity. PB may be present more often than suspected in adult congenital heart disease patients with predominant right ventricular (RV) dysfunction and have noteworthy prognostic implications.

It is well documented that left ventricular failure is associated with unstable cardiorespiratory control. ${ }^{1} \mathrm{~PB}$ is characterised by oscillations in the depth of ventilation (with or without apnoeas) paired with synchronous oscillations in heart rate and blood pressure with respect to the cardiovascular system. ${ }^{2}$ PB in patients with chronic heart failure (CHF) is a powerful predictor of poor outcome. Furthermore, treatment for $\mathrm{PB}$ improves both cardiac function and prognosis in these patients. ${ }^{1}$ Although theoretical mechanisms involving increased circulatory time and chemoreflex dysfunction have been proposed, the origin of PB remains a matter of debate. ${ }^{2}$ We have recently demonstrated features of compensated CHF among adult patients with congenital heart disease. ${ }^{3}$ There is no information at present whether PB is present in this rapidly expanding patient population, where RV dysfunction often predominates.

\section{METHODS}

We describe for the first time PB in two young adult patients with ToF 29 and 28 years after repair. Both patients were in New York Heart Association functional class II with body mass indices of $29.8 \mathrm{~kg} / \mathrm{m}^{2}$ and $41.5 \mathrm{~kg} / \mathrm{m}^{2}$, respectively. One patient had normal pulmonary function and the other moderately impaired pulmonary function as assessed by spirometry. Studies were performed with patients lying semirecumbent in standardised conditions in a quiet room. Breathing was recorded by strain gauge plethysmography (Hokanson EC4 plethysmograph). Oxygen saturation was measured with pulse oximetry (Nellcor 2000). Having diagnosed $\mathrm{PB}$, we investigated the potential pathophysiological substrate for PB (fig 1). Patients underwent chemoreflex testing to evaluate sensitivity and time delay as previously described. ${ }^{2}$ Gas exchange was monitored by a mass spectrometer (Amis 2000; Innovision). Cardiac output was measured by Doppler echocardiography and brain natriuretic peptide (BNP) concentrations were measured.

\section{RESULTS}

Results for the two subjects and corresponding normal values are shown in table 1 . Both patients demonstrated an attenuated breathing response to changes in $\mathrm{PCO}_{2}$, low cardiac output, and prolonged circulatory time. Patient 1
Table 1 Tests results in tetralogy of Fallot patients with periodic breathing.

\begin{tabular}{lccl}
\hline Parameters & Patient 1 & Patient 2 & Normal values \\
\hline MRI & & & \\
RVEDV (ml) & 198 & 378 & $88-227$ \\
RVESV (ml) & 108 & 233 & $23-103$ \\
RVEF (\%) & 46 & 38 & $47-80$ \\
RVMI (g/m ${ }^{2}$ ) & 66 & 105 & $<33$ \\
PRF (\%) & 40 & 40 & \\
LVEF (\%) & 62 & 53 & $56-78$ \\
Chemoreflex gain & 1.6 & 1.7 & $2.4(1.15)$ \\
(I/min/mm Hg) & & & \\
Time delay (min) & 0.43 & 0.36 & $0.3(0.04)$ \\
Cardiac output (l/min) & 2.8 & 3.2 & 4 \\
BNP (pmol/l) & 8.4 & 7.1 & $<4$ \\
\hline
\end{tabular}

BNP, brain natriuretic peptide; LVEF, left ventricular ejection fraction; PRF, pulmonary regurgitant fraction; RVEF, right ventricular ejection fraction; RVEDV, right ventricular end diastolic volume; RVESV, right ventricular end systolic volume; RVMI, right ventricular mass index.

had more impaired cardiac output and consequently a longer circulatory time delay than patient 2. In addition, both patients demonstrated increased BNP concentrations. Cardiovascular magnetic resonance was performed to assess pulmonary regurgitant fraction, ventricular volumes, mass, and ejection fraction. ${ }^{4}$ Both subjects had PR with dilated and hypertrophied right ventricles and moderate to severe impairment of RV function. Patient 2 also had mild left ventricular dysfunction.

\section{DISCUSSION}

PB occurs in adult congenital heart disease patients and relates to abnormal right sided haemodynamics. Abnormal chemoresponsiveness, long circulatory delay, and elevated sympathetic activity were all present in both patients, suggesting a common causality of PB in adult congenital heart disease patients and in patients with left heart failure. $\mathrm{PB}$ is associated with important cardiovascular complications, namely pulmonary vasoconstriction and increased RV afterload (caused by hypoxaemia), elevated sympathoadrenal activity (secondary to hypoxaemia or arousals from sleep), and prolonged circulatory time, possibly a reflection of decreased cardiac output. ${ }^{2}$ All these are of particular relevance to patients with ToF, whereas $\mathrm{PR}$ and its detrimental effects on RV function are the main causes of late morbidity and mortality. ${ }^{5}$ The combination of PB and RV dysfunction may have a negative synergistic effect. Even mild pulmonary vasoconstriction, secondary to hypoxaemia caused by PB, may augment PR and in turn exaggerate its detrimental effects on RV function and overall outcome late

Abbreviations: $\mathrm{BNP}$, brain natriuretic peptide; $\mathrm{CHF}$, chronic heart failure; $\mathrm{PB}$, periodic breathing; $\mathrm{PR}$, pulmonary regurgitation; $\mathrm{RV}$, right ventricular; ToF, tetralogy of Fallot. 

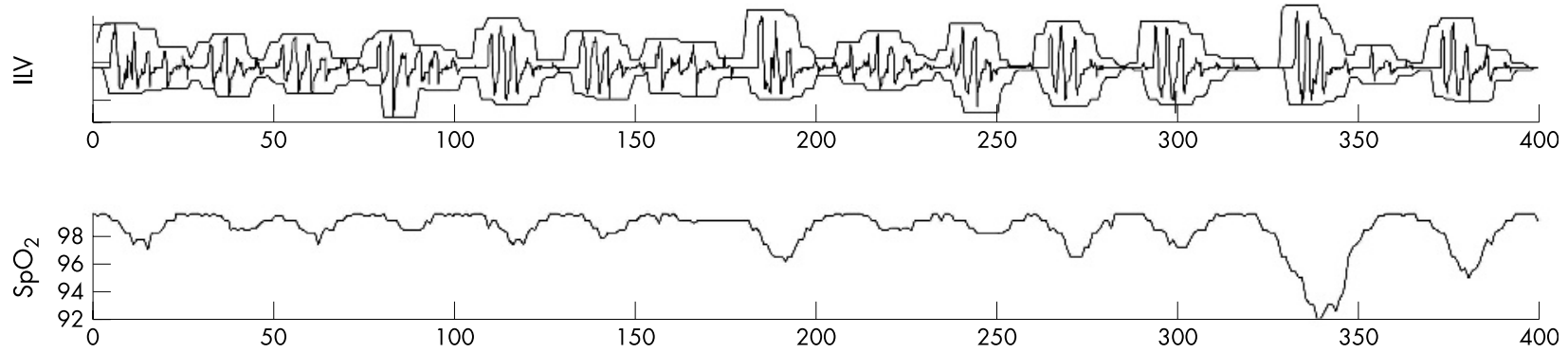

Figure 1 Short term resting recordings of respiratory movements (independent lung ventilation) and arterial oxygen saturation at the ear $\left(\mathrm{SpO}_{2}\right)$ from a patient with tetralogy of Fallot.

after repair. Neurohormonal dysregulation and autonomic nervous dysfunction, resulting from impaired right sided haemodynamics, ${ }^{4}$ may also be exacerbated by PB leading to an even worse outcome. ${ }^{5}$ Furthermore, PB and other abnormal pathways discussed here may represent suitable therapeutic targets, usually in combination with haemodynamic intervention. Whether $\mathrm{PB}$ and the resulting large synchronous oscillations in heart rate, blood pressure, and oxygen saturation have the same adverse prognostic implications in adult congenital heart disease as in left heart failure, remains to be elucidated.

\section{ACKNOWLEDGEMENTS}

P Georgiadou is supported by the Wellcome Trust and SV BabuNarayan is supported by the British Heart Foundation.

\section{Authors' affiliations}

P Georgiadou*, S V Babu-Narayan, D P Francis, M A Gatzoulis, Royal Brompton Hospital and National Heart and Lung Institute, Imperial College School of Medicine, London, UK

D T Kremastinos, Onassis Cardiac Centre, Athens, Greece
*Also Onassis Cardiac Centre, Athens, Greece

Correspondence to: Dr Panagiota Georgiadou, Onassis Cardiac Centre, 156 Syggrou Avenue, 17674 Athens, Greece; p.georgiadou@ imperial.ac.uk

Accepted 13 November 2003

\section{REFERENCES}

1 Kaneko Y, Floras J, Usui K, et al. Cardiovascular effects of continuous positive airway pressure in patients with heart failure and obstructive sleep apnea. N Engl Med 2003;348:1233-41.

2 Francis DP, Willson K, Davies LC, et al. Quantitative general theory of periodic breathing in chronic heart failure and its clinical implications. Circulation 2000;102:2214-21

3 Bolger AP, Sharma R, Li W, et al. Neurohormonal activation in adults with congenital heart disease: evidence of the chronic heart failure syndrome. Circulation 2002; 106:92-9.

4 Davlouros PA, Kilner P, Hornung TS, et al. Biventricular function in adults with repaired tetralogy of Fallot assessed with cardiovascular magnetic resonance: Detrimental role of right ventricular outflow aneurysms or akinesia and adverse right-to-left ventricular interaction. J Am Coll Cardiol 2002;40:2044-52.

5 Gatzoulis MA, Balaji S, Webber SA, et al. Risk Factors for arrhythmia and sudden cardiac death late after repair of tetralogy of Fallot: a multicentre study. Lancet 2000;356:975-81.

\section{IMAGES IN CARDIOLOGY}

\section{Left ventricular trabecular non-compaction}

A 41 year old man presented with transient atypical chest pains and was noted to meet criteria for left ventricular hypertrophy on his ECG. He therefore was referred for transthoracic echocardiography, which suggested some features of apical hypertrophic cardiomyopathy. In order to clarify this diagnosis, he was referred for a cardiovascular magnetic resonance scan, which revealed the presence of extensive fine trabeculation over the endocardial surface of the left ventricular myocardium, sparing only the septum. This may reflect embryonic persistence of fine trabeculation or non-compaction, most pronounced in the apical area where the myocardium is thinned. Non-compaction, in younger individuals, has been associated with a malignant outcome, but there is a paucity of data to suggest whether noncompacted trabeculation such as this has any adverse outcome. Our patient remains asymptomatic.
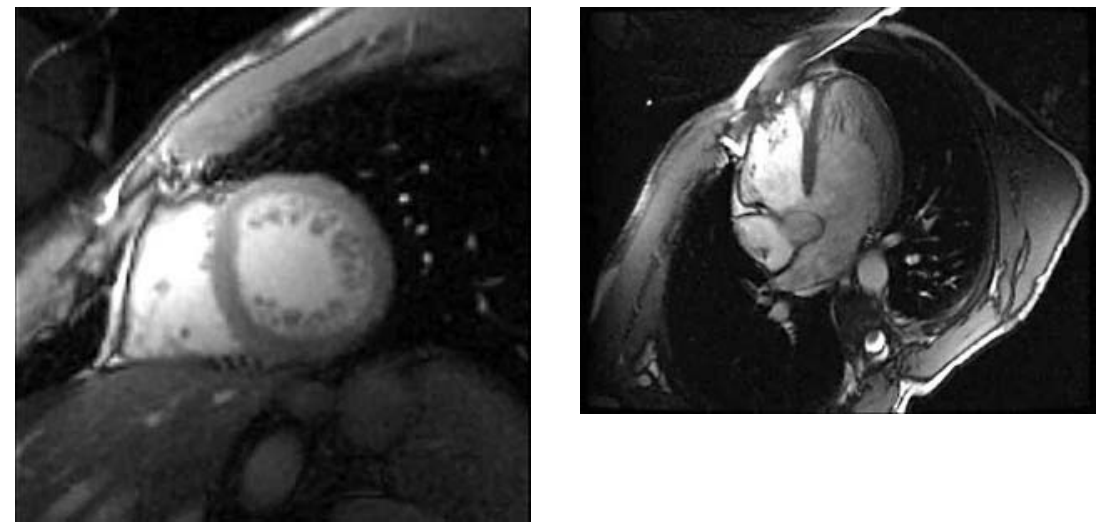

K C R Patel

D Pennell

F Leyva-Leon

drkiranpatel@hotmail.com 